\section{Realizing the potential of genomics: Translation is not translational research}

To the Editor:

Ne were delighted to read the recent review by Khoury et al. ${ }^{1}$ describing the Genomic Applications in Practice and Prevention Network. Translation of genomic medicine is an essential goal for the medical and public health communities, and we welcome GAPPNet as a laudable initiative to accelerate the effective transfer of emerging genomic knowledge and applications into improved clinical care. We agree with the authors' assertion that a coordinated system for translation can have a substantial impact on current barriers to progress in the translational process. However, we suggest that their model, although an excellent one, does not adequately address two key elements additionally required for optimal translation: recognition of the limitations of translational research and the need for explicit processes of policy and service development.

Recent years have heralded a growing commitment to translational research on both sides of the Atlantic, ${ }^{2}$ typically separated into two distinct categories: the translation of scientific understanding into a product or intervention and the translation of these new products into practice for patient benefit. ${ }^{3}$ It was perceived that greater attention to these two activities, referred to respectively as "from bench to bedside" and "research into practice," or as "T1" and "T2" research, could overcome delays in the translational process. ${ }^{4}$ This became the focus of a new "roadmap" by the National Institutes of Health. 5,6 The Cooksey Report, 7 a strategy for the funding of UK health research, similarly identified first and second gaps in translation, proposing that translational research would serve to plug them. More recently, Khoury further dissected the translational process, suggesting that T2 research could be subdivided into three phases of translation in addition to T1 research: T2, the evaluation and development of evidence-based guidelines; T3, research to move evidence-based guidelines into health practice; and $\mathrm{T} 4$, research to evaluate the health outcomes of a genomic application in practice. ${ }^{8}$

In practice, interest, activity, and funding are not evenly distributed along the translational pathway; US authors have shown how T1 research typically overshadows T2. ${ }^{3}$ Similarly, UK figures also show that the bulk of research funding goes on basic research and Type 1 translation. ${ }^{7}$ However, the paucity of funding for translational work is not the only obstacle to progress. Policy makers, academics, and research funders equate this need too tightly with the research paradigm and conflate translation with translational research. For example, the influential UK Genomic Medicine report is explicitly focused solely on the first of the two "gaps in translation" as discussed by Cooksey; it calls for the Office for the Strategic Co-ordination of Health Research to be charged with developing a strategic vision for translation, and the National Institute for Health Research with monitoring developments in genomic medicine and their implications for health services. ${ }^{8}$

GAPPNet builds very much on the T1-T4 model of translation; it seeks to collate information within key domains, which include translation research and translation programs. Although for the latter, there is recognition of the need for translation efforts "focused on the implementation of validated genomic applications in practice and prevention, improving health care quality, and reducing costs," we suggest that much of this activity as described is likely to take the form of applied health research, along with educational efforts. However, achieving translation of genomic medicine requires the movement of knowledge from the academic and research arena into a world of policy makers, clinical practitioners and health service managers, a community with different objectives, reward strategies, and culture. Research, whether basic or translational, is necessarily part of the academic paradigm. This is not to suggest that translational research of the type that GAPPNet seeks to support is not crucial, but rather that clinical translation will only be effective if mirrored by suitable developments in public policy and health services and that movement from research into practice requires explicit processes and activities to achieve this.

Policy development is complex, as is the integration of new interventions into the health care system, and relies on a pluralistic approach involving not only commissioners but also researchers and innovators along with potential health service deliverers, users, managers, and other stakeholders. The essential role of different communities for translation from knowledge to practice is widely acknowledged, ${ }^{9}$ but the complex and challenging nature of what public health genomics has termed "knowledge integration," 10 and the need for someone to specifically and explicitly drive the formulation of policy, rather less so.

The Canadian Health Services Research Foundation has defined this process of linking researchers and decision makers for policy development as knowledge brokering, ${ }^{11}$ also characterized as one of the missing links that can "bridge the know-do gap for health services."12 Effective knowledge brokering requires not only data for evidence-based assessment of the scientific and clinical merits of an innovation but also understanding and consideration of the priorities, concerns, and constraints of the wider stakeholders and the decision-making process. Policy development for genomic medicine requires adequate consideration of the societal context and the ethical and legal implications of innovations because these factors will be as important as the science itself in determining the success of their translation into clinical and public health interventions.

There can be little dissent with the assertion that prompt translation from research into health practice and thence into improved health outcomes is a wholly desirable end. Recognition of the need to devote resources and attention to realizing the practical benefits of biomedical research efforts is increasing but has not gone far enough. Translation as knowledge brokering and active policy development must be recognized as an explicit activity in its own right, as an essential final stage in moving the fruits of innovation into health services and public policy. We suggest that this previously overlooked component of translation merits considerably greater recognition and that dedicated organizations should be established for the specific purpose of mediating the efficient movement of knowledge into action to achieve better health. ${ }^{13}$

\section{Ron L. Zimmern, MA, FRCP, FFPHM Philippa C. Brice, MA, PhD PHG Foundation Cambridge, United Kingdom}

Both authors are affiliated with the PHG Foundation, an independent, multidisciplinary charitable organization that works to 
achieve the responsible and evidence-based translation of biomedical research for health. There are no commercial or competing interests to declare.

\section{REFERENCES}

1. Khoury MJ, Feero WG, Reyes M, et al. The genomic applications in practice and prevention network. Genet Med 2009;11:488-494.

2. Lenfant C. Shattuck lecture-clinical research to clinical practice-lost in translation? N Engl J Med 2003;349:868-874.

3. Woolf SH. The meaning of translational research and why it matters. JAMA 2008;299:211-213.

4. Sung NS, Crowley WF Jr, Genel M, et al. Central challenges facing the national clinical research enterprise. JAMA 2003;289:1278-1287.

5. Zerhouni EA. Translational and clinical science-time for a new vision. N Engl J Med 2005;353:1621-1623.

6. Zerhouni E. Medicine. The NIH Roadmap. Science 2003;302:63-72.

7. Cooksey D. A review of UK health research funding. London: HMSO, 2006.

8. House of Lords Science and Technology Committee. Genomic medicine. 2nd Report of 2008/09, HL Paper 107. 7-7-0009. London: The Stationery Office Limited.

9. Pronovost PJ, Berenholtz SM, Needham DM. Translating evidence into practice: a model for large scale knowledge translation. BMJ 2008:337:963965.

10. Genome-based research and population health. Report of an expert workshop held at the Rockefeller Foundation Study and Conference Center, Bellagio, Italy. 2006. Available at: http://www.phgfoundation.org/file_ admin/secure_file.php?file_ID=676. Accessed April 14-20, 2005.

11. Canadian Health Services Research Foundation. Knowledge Brokering. 2009. Available at: http://www.chsrf.ca/keys/use_knowledge_e.php. Accessed August $25,2009$.

12. Lomas J. The in-between world of knowledge brokering. BMJ 2007;334: $129-132$.

13. van $\mathrm{KJ}$, de $\mathrm{SD}$, Sewankambo N. Using knowledge brokering to promote evidence-based policy-making: the need for support structures. Bull World Health Organ 2006;84:608-612.

\section{Translation research is an essential but not sufficient ingredient for translation of genomic medicine into population health benefits}

\section{To the Editor:}

appreciate and agree with the comments of Zimmern and Brice ${ }^{1}$ on the recent article describing the Genomic Applications in Practice and Prevention Network. ${ }^{2}$ Translation of genomic advances into population health benefits is a shared goal for the medical and public health communities. The premise of GAPPNet is that translation research, such as comparative effectiveness research, ${ }^{3}$ will make translation more evidence based. Translation research is a necessary but not sufficient ingredient for translation. From other fields of medicine, we know that many evidence-based applications seem to be "lost in translation" and do not make the desired impact on population health. ${ }^{4}$ GAPPNet will address translation in the context of translation research and the need for explicit processes of guidelines, policy, and service development. One of the four components of GAPPNet are nonresearch translation programs such as the ones that Centers for Disease Control and Prevention recently funded. ${ }^{5}$ I agree that "achieving translation of genomic medicine requires the movement of knowledge from the academic and research arena into a world of policy makers, clinical practitioners and health service managers, a community with different objectives, reward strategies and culture." As a stakeholder-driven enterprise, GAPPNet will attempt to do the kind of knowledge brokering that the authors allude to ${ }^{6-8}$ by bringing the stakeholders together to consider their priorities, concerns, and constraints, in the context of social, ethical, legal, and financial implications of innovations. I look forward to an active GAPPNet stakeholder process that should be enhanced by translation research and evaluation, leading to appropriate use of genomic knowledge for the benefit of population health.

Muin J. Khoury, MD, PhD

Office of Public Health Genomics

Centers for Disease Control and Prevention

Atlanta, Georgia

Disclosure: The author declares no conflict of interest.

\section{REFERENCES}

1. Zimmern RL, Brice PC. Realizing the benefits: translation is not translation research. Genet Med 2009;11:897-898.

2. Khoury MJ, Feero WG, Reyes M, et al. The Genomic Applications in Practice and Prevention. Genet Med 2009;11:488-494.

3. Khoury MJ, Rich EC, Randhawa G, Teutsch SM, Niederhuber J. Comparative effectiveness research and genomic medicine: an evolving partnership for 21sy century medicine. Genet Med 2009;11:707-711.

4. Lenfant C. Shattuck lecture-clinical research to clinical practice-lost in translation? N Engl J Med 2003;349:868-874.

5. Centers for Disease Control and Prevention. The Genomic Applications in Practice and Prevention Network. Available at: http://www.cdc.gov/genomics/ translation/GAPPNet/index.htm. Accessed September 17, 2009.

6. Pronovost PJ, Berenholtz SM, Needham DM. Translating evidence into practice: a model for large scale knowledge translation. BMJ 2008;337:963965.

7. Canadian Health Services Research Foundation. Knowledge Brokering. 2009. Available at: http://www.chsrf.ca/keys/use_knowledge_e.php. Accessed September 15, 2009.

8. van KJ, de SD, Sewankambo N. Using knowledge brokering to promote evidence-based policy-making: the need for support structures. Bull World Health Organ 2006;84:608-612. 